\title{
Flanker recall and the flanker validity effect may reflect different attentional processes
}

\author{
PATRICIA A. SCHMIDT and VERONICA J. DARK \\ Iowa State University, Ames, Iowa
}

\begin{abstract}
High flanker recall in the correlated flanker task suggests a failure of selective attention. We used a dissociation approach to examine the role of attentional processing of the flankers' identities in the flanker validity effect (FVE). In three experiments, flanker recall decreased as flanker duration decreased, but the FVE was not affected by flanker duration. Masking also produced a dissociation, in that masking decreased flanker recall but the FVE was not affected. Examination of on-line flanker identification suggested that flanker recall was an adequate measure of attentional processing of the flankers' identities on most trials. The results suggest that attentional processing of the flankers' identities is not related to the magnitude of the FVE and is not necessary for it to obtain. Because there is a failure of selective attention, the dissociations suggest that flanker recall and the FVE may reflect different attentional processes.
\end{abstract}

Eriksen and colleagues (B. A. Eriksen \& C. W. Eriksen, 1974; C. W. Eriksen \& Hoffman, 1972, 1973) developed the flanker task to examine the extent to which to-be-ignored flanking letters would influence the binary response to a target letter that occurred at a fixed location. The task was assumed to involve focused attention. Results showed that flankers influenced responses: When the flankers were letters that had been assigned to the same response as the target (compatible trials), response times were faster than when the flankers were letters that had been assigned to the other response (incompatible trials). The Eriksen flanker effect has been used as supporting evidence for the late-selection view of attention, which states that processing of stimulus identities is automatic (e.g., Cowan, 1988; C. W. Eriksen \& Hoffman, 1973; Shiffrin, Diller, \& Cohen, 1996). However, this interpretation has been challenged by proponents of the early-selection view of attention on the basis of evidence suggesting a failure of selective attention in the flanker task (see Johnston \& Dark, 1986; Yantis \& Johnston, 1990).

Evidence that attention is not focused on just the target in the flanker task comes from studies demonstrating that the magnitude of the Eriksen flanker effect depends on display characteristics related to the ease of target selection. For example, increasing target-flanker separation has been shown to reduce the Eriksen flanker effect (B. A. Eriksen \& C. W. Eriksen, 1974), even when visual

Portions of the data were presented at the 67th and 69th meetings of the Midwestern Psychological Association in Chicago, May 1995 and 1997. We thank Karla Benson, Brian Crabb, Heidi Faye, Ting-Jung Hsu, James Stamper, and Steve Willis for help with data collection. Correspondence concerning this article should be sent to either P. A. Schmidt, Department of Psychology, University of Michigan, Flint, MI 48502, or V. J. Dark, Department of Psychology, Iowa State University, Ames, IA 50011-3180 (e-mail: schmidt@flint.umich.edu or vjdark@ iastate.edu). acuity is controlled (Miller, 1991). Similarly, there is a reduction in the Eriksen flanker effect when the target is made featurally distinct from the flankers, either by presenting the target in one color and the flankers in another color (Harms \& Bundesen, 1983) or by using displays that allowed the target and flankers to be perceived as different objects as a result of Gestalt grouping principles (Kramer \& Jacobson, 1991). The reduction in the magnitude of the Eriksen flanker effect was interpreted by early-selection theorists as evidence that a failure of selective attention is necessary for the Eriksen flanker effect. However, late-selection theorists countered that the reduction of the Eriksen effect shows only that the effect may be sensitive to manipulations of attention, so that some attentional processing enhances it (cf. the partially automatic processes of Kahneman \& Treisman, 1984); the failure of such manipulations to eliminate the effect suggests that attention is not necessary for the Eriksen flanker effect. Thus, although there may be a failure of selective attention in the Eriksen flanker task, the extant data do not demonstrate that attentional processing of the flankers is involved in producing the Eriksen flanker effect.

One reason suggested for the failure of selective attention in the Eriksen flanker task was that the flankers, because they sometimes served as targets, are atypical, or primed, stimuli that may attract attention (see, e.g., Johnston \& Dark, 1986; Kahneman \& Treisman, 1984). Miller (1987) developed the correlated flanker task as an alternative focused-attention task that would not be subject to such failures of selective attention, because the flankers were not primed. In the correlated flanker task, target letters never appear in the flanker locations, and flanker letters never appear in the target location; that is, stimuli are consistently mapped to location (cf. Shiffrin \& Schneider, 1977). However, in the correlated flanker task, the to-be-ignored flankers can become associated with responses because they co-occur regularly with targets re- 
quiring a specific response--that is, flanker-response correlations exist.

Of interest in the correlated flanker task are responses to two types of trials: valid trials, in which the flankers are highly correlated with the same response as that required by the target (and, therefore, validly cue the target response), and invalid trials, in which the flankers are highly correlated with the other response (and, therefore, invalidly cue the target response). Research with the correlated flanker task has shown that response times are slowed to targets on invalid trials, relative to valid trials (e.g., Carlson \& Flowers, 1996; Miller, 1987, 1991; Paquet \& Lortie, 1990; Schmidt \& Dark, 1998; Stadler \& Proctor, 1993). That is, response times showed that participants are influenced by the flanker-response correlations. Miller (1987) labeled this phenomenon the flanker validity effect (FVE) and suggested that it reflected the influence of the identities of automatically processed, unprimed, unattended flankers on target processing. Further support for automatic processing of the flankers' identities came from the fact that participants were not able to recall the flankers at the end of the experiment or to describe the flanker-response correlations (Miller, 1987).

Several investigators have challenged Miller's lateselection interpretation of the FVE in the correlated flanker task. Paquet and Lortie (1990) found that cuing the target location (even though the target occurred in a fixed location) reduced the FVE. They used the argument previously applied to the Eriksen flanker task: Because a factor that allowed participants to focus their attention on just the target (i.e., location cuing) reduced the magnitude of the FVE, the FVE might be reflecting attentional processing of the flankers' identities that occurs because of a failure of selective attention (see, also, Stadler \& Proctor, 1993). Schmidt and Dark (1998) offered further support for this interpretation. They used flanker recall as a measure of the attentional processing of the flankers' identities (cf. Fisk \& Schneider, 1984; Roediger, 1990; Shiffrin \& Schneider, 1977). Unlike Miller (1987), who showed no reliable flanker recall, Schmidt and Dark found high levels of flanker recall. Schmidt and Dark reconciled the conflicting outcomes by examining differences in the ways in which recall was assessed and differences in the experimental contexts in which recall was assessed. They argued that their forcedresponse recall measure was more sensitive to attentional processing of the flankers than Miller's open-ended measure. Thus, they interpreted their high flanker recall as evidence that there was a failure of selective attention at some point in the processing in the correlated flanker task.

The high flanker recall reported by Schmidt and Dark (1998) indicated that the identities of flankers in the correlated flanker task are attentionally processed, but it is not clear from the extant data whether the attentional processing of the flankers' identities is related to the FVE. The fact that flankers are receiving sufficient levels of attentional processing to be identified on a latter recall test does not, in itself, imply that the FVE depends on this processing. As was pointed out by Jeff Miller in a review of an earlier version of this paper, it is possible that automatic processing of the flankers' identities both influences target processing, producing the FVE, and leads to the formation of a memory trace of the flankers. When target processing is complete, attentional processing may be directed to the memory trace of the flankers, and it could be attentional processing of this trace that produces high flanker recall. Thus, although a suggestive finding reported by Schmidt and Dark was the small, but reliable, correlation between the magnitude of the FVE and level of flanker recall, the data showed only that reliable flanker recall and a reliable FVE co-occurred.

The main issue in the early- versus late-selection controversy is whether stimulus identity is processed automatically or whether it requires attention. In order to understand whether attentional processing of flanker identities plays a necessary role in the flanker effect, it must be determined whether the flanker effect occurs under conditions in which there is no attentional processing of the flankers' identities. As long as there is a failure of selective attention, the situation is ambiguous. The purpose of the current research was to examine the role of attentional processing of the flankers' identities in the FVE. From the perspective of late selection, attentional processing of flanker identity is not necessary for the FVE, only automatic processing of the identities is needed. From the perspective of early selection, attentional processing of flanker identity is necessary for the FVE. Although the two approaches differ in terms of which type of processing of flanker identity, attentional or automatic, is responsible for the FVE, flanker recall is assumed to reflect attentional processing of flanker identity according to both approaches. Thus, the question of interest was whether flanker recall and the FVE reflect the same type of processing of flanker identities or whether they reflect two different types of processing.

We employed a dissociation logic to investigate the question. To the extent that flanker recall reflects attentional processing and the FVE reflects automatic processing of the flankers' identities, only flanker recall should be influenced by a variable that affects attentional processing; that is, there should be a functional dissociation. However, if there is no functional dissociation and both flanker recall and the FVE are influenced similiarly, there is evidence that both flanker recall and the FVE reflect a common stage of attentional processing.

\section{EXPERIMENT 1}

Experiment 1 was designed to assess changes in the FVE as a function of variations in attentional processing of the identities of the flankers. To vary attentional processing of flanker identity, we manipulated flanker duration. We presented targets for $500 \mathrm{msec}$ with flankers that had a duration of $500,400,300,200$, or $100 \mathrm{msec}$. The longer flanker durations were assumed to be sufficient to support both automatic and attentional process- 
ing of flanker identity. Because flanker recall was assumed to reflect the amount of attentional processing of flanker identity, we expected that flanker recall would decrease monotonically as flanker duration decreased. We expected there to be so little attentional processing of flanker identities at the shorter durations that flanker recall would be at or near chance. By examining the relationship between the level of flanker recall and the magnitude of the FVE, we could determine whether both were influenced in the same way by the duration manipulation or whether there was a dissociation.

\section{Method}

\section{Participants}

One hundred forty right-handed students (40 male and $100 \mathrm{fe}$ male) received extra credit in an undergraduate psychology course for participating in this experiment. The participants were assigned to one of the five flanker duration conditions $(500,400,300,200$, and $100 \mathrm{msec}$ ). Each participant was tested in a single session lasting approximately $45 \mathrm{~min}$. The data from 4 participants ( 2 in the $400-$ msec condition, 1 in the 200 -msec condition, and 1 in the $100-\mathrm{msec}$ condition) with a target-response accuracy of less than $85 \%$ correct were discarded and replaced with data from new participants.

\section{Apparatus and Stimuli}

Stimulus presentation and response recording were controlled by a Zenith-386 computer programmed with the MicroExperimental Laboratory (MEL) software (Schneider, 1988). The stimuli were strings of three letters from the standard set of alpha-numeric characters, presented in a row in the center of a VGA computer monitor. A blank space separated each letter. Letters were approximately $0.4 \mathrm{~cm}$ wide and $0.5 \mathrm{~cm}$ high. Displays were viewed from approximately $60 \mathrm{~cm}$. The display parameters were such that the distance from the center of the target to the center of each flanker was about $0.8^{\circ}$ of visual angle horizontally.

\section{Procedure}

Each participant was randomly assigned eight letters from the consonants B-W. Two letters were targets requiring an index-finger response, two were targets requiring a middle-finger response, and the remaining four were flankers. The " $\mathrm{N}$ " key was used for rightindex-finger responses and the " $M$ " key was used for right-middlefinger responses. Flanker validity was defined in terms of the percentage of trials in which a given flanker appeared with a given target. Flanker validity was $80 \%$; that is, two of the flankers appeared with index-finger targets on $80 \%$ of the experimental trials (valid trials) and with the middle-finger targets on $20 \%$ of the experimental trials (invalid trials). The other two flankers appeared with the middle-finger targets on $80 \%$ of the trials and with the index-finger targets on $20 \%$ of the trials. There were eight different valid and eight different invalid stimulus displays.

There was a total of 352 trials. The first 32 trials were practice trials, in which flanker validity was $100 \%$ (i.e., there were no invalid trials); each valid display was presented four times. Practice trials were not included in any analyses. The remaining 320 trials were the experimental trials, in which response times to valid and invalid trials were measured: Because flanker validity was $80 \%$, there were 256 valid trials and 64 invalid trials. The order of trials was random, with the constraint that, within each set of 40 trials, all 8 valid trials were presented four times each and each invalid trial was presented one time.

All the trials began with a 1,000-msec READY signal, in the center of the screen, and a location cue $\left(^{\wedge}\right)$ that appeared below the $A$ in READY. A 500-msec blank followed the READY signal, after which the stimulus display appeared in the center of the screen. Targets were presented for $500 \mathrm{msec}$. Responses could be made while the target was presented or during the 1,000-msec blank following target offset. Target-flanker onset was simultaneous, but target-flanker offset varied because of the flanker duration manipulation. For example, in the 300-msec flanker duration condition, the target and flankers were presented simultaneously; however, after $300-\mathrm{msec}$, the flankers were removed by presenting blanks in their place. As a result, the participants saw only the target during the last $200 \mathrm{msec}$ of the display.

The participants were instructed to respond to the target letter, which was the middle letter and which appeared above the place at which the location cue $\left(^{\wedge}\right)$ in the READY display had been. The participants also were instructed to ignore the flanking letters that were presented to the right and left of the target letter on each trial. The participants rested their index and middle fingers on the " $N$ " and " $M$ " keys throughout the experiment. Error feedback was presented immediately: Incorrect responses to targets and failures to respond within $1,500 \mathrm{msec}$ (target duration plus posttarget blank) were signaled by a $500-\mathrm{msec} 400-\mathrm{Hz}$ tone. The intertrial interval was $1,000 \mathrm{msec}$, during which the computer screen was blank. The participants were given three self-terminated rest periods.

After the experimental trials, the participants were asked to verbally report the four target letters and the four flanker letters (flanker recall). ${ }^{1} \mathrm{~A}$ forced-recall procedure was used, so that, even when the participants were not confident that they could recall the flankers' identities, they were required to report four flanker letters. Finally, all the participants were debriefed, and any questions they had were answered before the experimental session was terminated.

\section{Results}

An alpha level of .05 was used for all analyses.

\section{The Flanker Validity Effect}

A separate mean response time was computed for valid and for invalid trials for each participant; only accurate response times were included. The group mean response times and proportions correct for valid and invalid trials for the five flanker durations are shown in Table 1 . In both validity conditions across all durations, accuracy was above $95 \%$. In general, higher accuracy was associated with faster response times, so there was no evidence of speed-accuracy tradeoffs.

An analysis of variance (ANOVA) performed on the response times, with duration $(500,400,300,200$, and $100 \mathrm{msec}$ ) as a between-subjects variable and validity (valid and invalid) as a within-subjects variable, showed a reliable effect of validity $\left[F(1,135)=36.86, M S_{\mathrm{e}}=\right.$ 182.2], demonstrating the FVE in which response times are faster for valid trials than for invalid trials. Neither

Table 1

Mean Response Time (in Milliseconds) With Its Standard Deviation and Proportion Correct for Invalid and Valid Trials as a Function of Flanker Duration in Experiment 1

\begin{tabular}{cccccccc}
\hline & \multicolumn{5}{c}{ Trial Type } \\
\cline { 2 - 5 } \cline { 5 - 7 } $\begin{array}{c}\text { Flanker Duration } \\
\text { (msec) }\end{array}$ & \multicolumn{3}{c}{ Invalid } & & \multicolumn{3}{c}{ Valid } \\
\cline { 2 - 7 } \cline { 5 - 7 } & $M$ & $S D$ & PC & & $M$ & $S D$ & PC \\
\hline 500 & 561 & 82 & .96 & & 549 & 79 & .97 \\
400 & 545 & 76 & .95 & & 535 & 78 & .96 \\
300 & 542 & 68 & .95 & 535 & 74 & .97 \\
200 & 541 & 73 & .96 & 534 & 71 & .96 \\
100 & 530 & 64 & .96 & 517 & 62 & .96 \\
\hline
\end{tabular}


the effect of duration nor the duration $x$ validity interaction effect was reliable (both $F \mathrm{~s}<1$ ).

Although the duration $X$ validity interaction was not reliable, the reliability of the FVE was examined separately for each duration. The FVE was computed as the difference between the response times on invalid and valid trials for each duration and is plotted in Figure 1. One-tailed comparisons against zero were used to determine whether the observed FVEs were reliably greater than chance. The FVE was reliable in all duration conditions except the 200-msec condition, which did approach reliability $(.05<p<.10)$. A power analysis examining the effect of duration on the magnitude of the FVE revealed that the analysis had enough power to detect an effect size of 0.30 (a relatively conservative effect size; Cohen, 1988) with .80 probability. Thus, it appears that flanker duration does not have a strong effect on the magnitude of the FVE.

\section{Flanker Recall}

The mean proportions of flankers correctly recalled are shown in Figure 2 as a function of duration. A one-way ANOVA performed on flanker recall revealed a reliable effect of duration $\left[F(4,135)=5.66, M S_{\mathrm{e}}=0.07\right]$. A planned linear contrast was reliable $\left[F(1,134)=21.99, M S_{\mathrm{e}}=\right.$ $0.07]$, and the residual sum of squares was not. The contrast showed that, as flanker duration decreased in increments of $100 \mathrm{msec}$, the level of flanker recall also decreased monotonically.

Flanker recall in all five conditions also was compared to chance-level recall in order to determine whether the participants had memory of the flankers' identities across the duration conditions. Chance-level flanker recall, as computed with a hypergeometric distribution (with 22 letters, the alphabet minus four targets, sampled without replacment), was a proportion of .18 , or 0.73 flankers out of four. Flanker recall was reliably greater than chance in all five duration conditions. It appears that the current display parameters, although increasingly data limited, still provided enough information to support above-chance memory of the flankers' identities, as measured by recall, at all durations.

\section{Discussion}

Experiment 1 was designed to examine the role of attentional processing of the flankers' identities in the FVE. Attentional processing of the flankers' identities was manipulated by varying flanker duration. We reasoned that the data limitations brought about by brief presentation of the flankers would limit the amount of attentional processing of the flankers' identities. Because flanker recall reflects attentional processing of the flankers' identities, we expected flanker recall to decrease as a function of flanker duration and to reach chance level. As was predicted, the results showed a monotonic relationship between flanker recall and flanker duration, with longer durations associated with higher flanker recall. However, the display conditions employed failed to eliminate attentional processing of the flankers' identities: Flanker recall was above chance at all five flanker durations.

The magnitude of the FVE was relatively small, as compared with those reported by Miller (1987) and Paquet and Lortie (1990). It is likely that this reflects either the fact that we used more data-limited displays or the

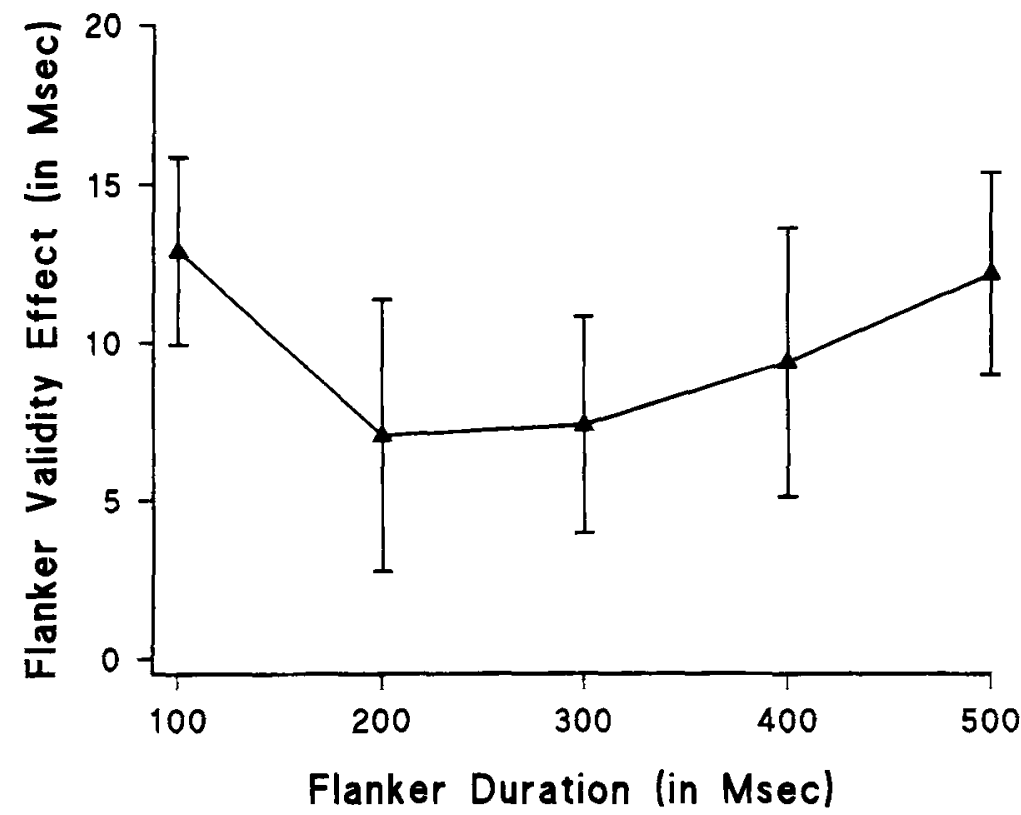

Figure 1. Mean flanker validity effect (in milliseconds) with standard error as a function of flanker duration in Experiment 1. 


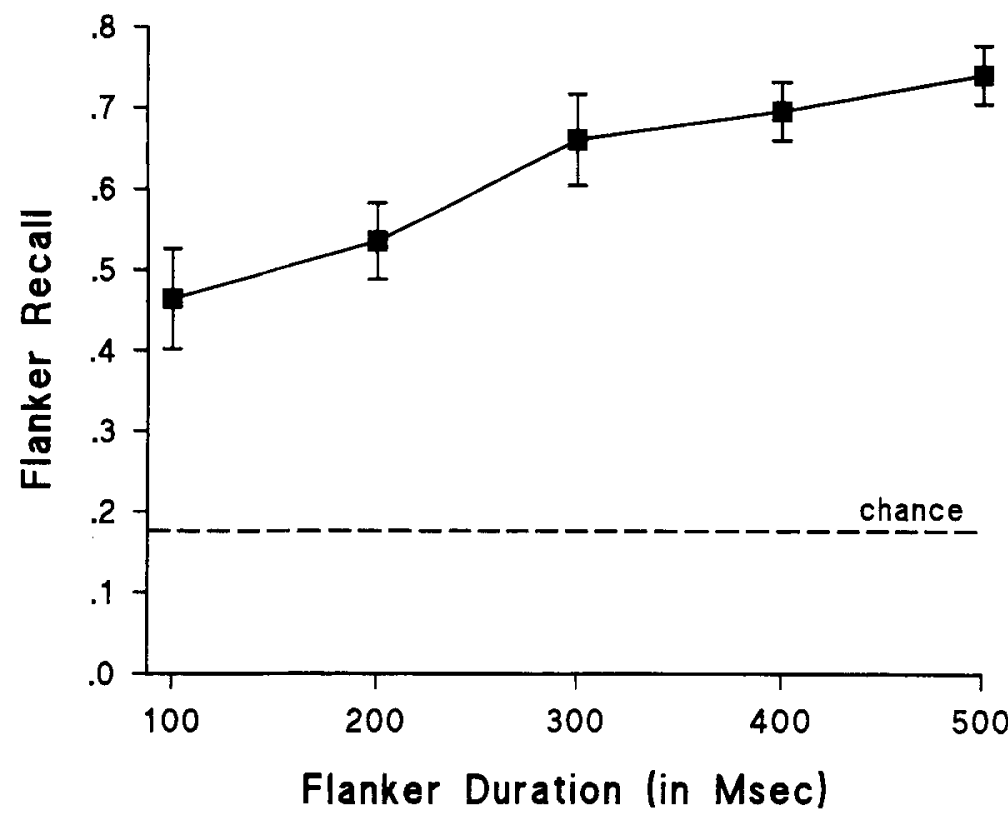

Figure 2. Mean proportion correct flanker recall with standard error as a function of flanker duration in Experiment 1.

fact that we used a lower level of validity. ${ }^{2}$ Our question was whether the FVE depends on attentional processing of the flankers' identities. To the extent that it does, the FVE should be influenced by the flanker duration manipulation. The FVE was reliable in all but the $200-\mathrm{msec}$ duration condition and did not vary reliably in magnitude across flanker durations. Thus, the results of Experiment 1 demonstrated a dissociation in the effect of duration on the FVE and flanker recall. Flanker recall remained above chance at all durations and decreased monotonically as a function of flanker duration. Although the FVE also was above chance at most durations, it did not vary as a function of flanker duration. The pattern of results suggested that more attentional processing of the flankers' identities does not increase the FVE.

There are at least two explanations for the dissociation outcome. The first is the late-selection explanation: The processing that leads to flanker recall is different from the processing that leads to the FVE; the dissociation reflects the fact that automatic processing of flanker identity is responsible for the FVE whereas attentional processing of flanker identity is responsible for flanker recall. The caveat to such a conclusion is that, although there is no relationship between amount of attentional processing of flanker identity, as measured by flanker recall, and the magnitude of the FVE, one cannot rule out the possibility that some minimal attentional processing of flanker identity is necessary for the FVE, because recall was above chance in all conditions. What is known is that more attentional processing of flanker identity does not increase the FVE.

A second possible explanation of the dissociation centers on interpretation of the flanker recall measure.
Schmidt and Dark (1998) argued that flanker recall was a sensitive measure of trial-by-trial attentional processing of flanker identity, and they assumed that assessing flanker recall near the end of the experiment successfully tapped into the sum of attentional processing of the flankers' identites over all trials. If, however, flanker recall, a discrete measure obtained at the end of the trials, only reflects the attentional processing of flankers' identities on an atypical subset of the trials, flanker recall may not provide information about attentional processing on the majority of trials (i.e., the trials that contribute to the FVE). As was pointed out by reviewers, ${ }^{3}$ high flanker recall could occur on the basis of just a few trials (e.g., the first few or the last few, or trials on which errors were made), and attentional processing of the flankers' identities on those trials might not be typical of the trials contributing to the FVE. If this were true, the dissociation would be uninformative as to whether the processes that lead to flanker recall and the FVE are the same or different.

The results of Experiment 1 revealed a functional dissociation in the effect of flanker duration on flanker recall and on the FVE. The remaining experiments were designed to extend and clarify the pattern of results obtained in Experiment 1 by ruling out competing explanations of the dissociation. Experiment 2 investigated whether flanker recall actually reflects the processing of flanker identities on most trials or whether it reflects processing on an atypical subset of the trials: Flanker recall after a series of trials was compared with on-line flanker identification. If flanker recall reflects something other than attentional processing of the flankers' identities on most trials, the dissociation becomes uninformative about the nature of typical flanker processing. Experi- 
ments 3 and 4 assessed the extent to which some minimal amount of attentional processing of the flankers' identities may be required to produce the FVE by employing brief flanker durations and backward masks. At issue was whether the FVE is eliminated under conditions that limit awareness of the flankers' identities.

\section{EXPERIMENT 2}

One of the benefits of assessing attentional processing of the flankers' identities with flanker recall is that participants are not alerted to the fact that the flankers' identities might be "important" until after the trials in which the FVE is assessed. Thus, flanker recall provides an uncontaminated estimate of attentional processing of the flankers' identities under standard instructions. This benefit of flanker recall also is its drawback: The fact that flanker recall is assessed near the end of the experiment makes it difficult to determine whether flanker recall reflects processing on almost every trial or whether it reflects processing on just a few trials, perhaps the first few or the last few, or trials on which errors were made.

An on-line measure of trial-by-trial flanker awareness could assess the extent of attentional processing of the flankers' identities throughout the experiment. Of course, the problem with assessing awareness of the flankers' identities during the experimental trials is that participants' strategies may change in such a way that they attempt to engage in more processing of the flankers' identities (cf. Rock, Linnett, Grant, \& Mack, 1992). If participants attempt to do more flanker processing, an online measure of attentional processing of the flankers' identities may overestimate attentional processing under the standard conditions that are of interest (when participants are instructed to ignore the flankers). In the current experiment, awareness of the flankers' identities on a given trial was assessed. To the extent that on-line report of flankers' identities does not cause a shift in the participants' strategies, and to the extent that report of flankers' identities mirrors flanker recall, there will be support for the idea that flanker recall does reflect processing of the flankers' identities on the typical trial.

Two steps were taken to reduce the likelihood of strategy changes in Experiment 2. First, the participants were trained with the standard procedure from Experiment 1 . After the training block, flanker recall was assessed. Second, on-line flanker identification was assessed on a random subset of trials, rather than on every trial. If the participants could report the identity of flankers on line, and if the FVE was found, there would be evidence that the participants were aware of the flankers' idenitities on trials that contributed to the FVE. The purpose of Experiment 2 was to determine whether flanker recall is a sensitive measure of the processing of flanker identity on a trial-by-trial basis. We employed the endpoint conditions from Experiment 1 (viz., the 500- and 100-msec durations) in this two-part experiment.

\section{Method}

\section{Participants}

Fifty-six right-handed students ( 22 male and 34 female) were recruited from the same participant pool as that in Experiment 1 and were given course credit in return for their participation. The participants were assigned to either the 500 - or the 100 -msec duration condition. Each participant was tested in a single session lasting approximately $35 \mathrm{~min}$. The data from 4 participants ( 2 from each duration condition) were replaced because of low target-response accuracy (less that $85 \%$ correct on valid trials).

\section{Procedure}

The apparatus and stimuli for Experiment 2 were identical to those for the corresponding duration conditions of Experiment 1. Experiment 2 consisted of two blocks of trials. Block 1 included 32 practice trials in which validity was $100 \%$ and 120 experimental trials in which validity was $80 \%$, and display randomization over each 40 trials was the same as that described for Experiment 1. The instructions for Block 1 were identical to the instructions given to the participants in Experiment 1. Following Block 1, the participants were asked to recall the index- and middle-finger response targets and the four flankers. As in Experiment 1, a forced-recall procedure was used that required the participants to report four flanker letters.

After assessment of flanker recall, the instructions for Block 2 were given. The participants were told that during the next block of trials, occasionally after responding to a target, they would be asked to report the identity of the flankers that had appeared with that target. The participants were instructed to continue to do the target discrimination task just as they had been doing it up to this point, but they also were instructed that whenever they saw a "What were the flankers?" prompt, their task was to type in the letter that they believed had been the flanker on the immediately preceding trial. We refer to these trials as the flanker identification trials.

Block 2 was composed of four types of trials: experimental trials, test trials, flanker identification trials, and buffer trials. There were 240 experimental trials, identical in the distribution of invalid and valid trials to the experimental trials in Block 1 . Embedded between every run of 6-10 experimental trials was one of 32 triplets, each consisting of a test trial, a flanker identification trial, and a buffer trial, in that order. The test trials were simply the trials for which awareness of the flankers was assessed. Each of the eight valid and eight invalid displays was presented twice as a test trial. Each test trial was followed by a flanker identification trial in which the "What were the flankers?" prompt was displayed until a reponse was made. Buffer trials occurred next; they were not included in any analyses. From the participant's point of view, only the flanker identificaiton trials were different.

\section{Results}

An alpha level of .05 was used for all analyses.

\section{Flanker Validity Effect}

A separate mean response time was computed for valid and for invalid trials for each participant; only accurate response times were included. The group mean response times and proportions correct for valid and invalid trials as a function of block (Block 1 and Block 2) and duration (500 and $100 \mathrm{msec}$ ) are shown in Table 2. For both durations, target response accuracy was above $94 \%$. An ANOVA on response times, with duration as a between-subjects variable and block and validity as withinsubjects variables, showed a reliable effect of block 
Table 2

Mean Response Time (in Milliseconds) With Its Standard Deviation and Proportion Correct for Invalid and Valid Trials as a Function of Flanker Duration and Block in Experiment 2

\begin{tabular}{|c|c|c|c|c|c|c|}
\hline \multirow[b]{3}{*}{ Condition } & \multicolumn{6}{|c|}{ Trial Type } \\
\hline & \multicolumn{3}{|c|}{ Invalid } & \multicolumn{3}{|c|}{ Valid } \\
\hline & $M$ & $S D$ & $\mathrm{PC}$ & $M$ & $S D$ & $\mathrm{PC}$ \\
\hline \multicolumn{7}{|c|}{$500-\mathrm{msec}$} \\
\hline Block 1 & 559 & 95 & .94 & 550 & 92 & .95 \\
\hline Block 2 & 619 & 111 & .95 & 603 & 98 & .95 \\
\hline \multicolumn{7}{|c|}{$100-\mathrm{msec}$} \\
\hline Block 1 & 528 & 72 & .96 & 520 & 73 & .95 \\
\hline Block 2 & 595 & 119 & .92 & 573 & 105 & .95 \\
\hline
\end{tabular}

$\left[F(1,54)=36.64, M S_{\mathrm{e}}=5,245.9\right]$, with faster response times for Block 1 , and a reliable effect of validity $\left[F(1,54)=26.49, M S_{\mathrm{e}}=397.6\right]$, with faster response times for valid trials reflecting the FVE. There also was a reliable block $\times$ validity interaction $[F(1,54)=4.02$, $\left.M S_{\mathrm{e}}=382.4, p=.05\right]$, reflecting a larger FVE in Block 2 . Neither the main effect of duration nor any of the other interactions was reliable (all $F_{\mathrm{s}}<1.5$ ).

The mean FVE was computed for Block 1 and Block 2 at each duration and was compared with zero, to determine whether the observed FVE was reliably greater than chance. The FVE was reliable for all combinations of block and duration.

\section{Knowledge of Flanker Identity}

Flanker recall. The mean proportion of flankers correctly recalled after Block 1 was $.45(S D=.27)$ for the 100 -msec condition and .62 (SD $=.24)$ for the $500-\mathrm{msec}$ condition. A comparison between the means revealed higher flanker recall in the $500-\mathrm{msec}$ than in the $100-\mathrm{msec}$ condition. Flanker recall was greater than chance (.18) in both conditions.

Flanker identification. The mean proportions of flankers correctly reported immediately after the valid and invalid test trials in Block 2 for the 100- and 500-msec conditions are shown in Table 3. An ANOVA on flanker identification, with duration and validity as variables, showed no reliable main effects and no interaction (all $F_{\text {s }}<1$ ). The mean flanker identification (collapsed over validity) from each duration was greater than chance (using .18 as a liberal estimate, which assumes that participants do not report the targets as flankers), providing evidence of attentional processing of the flankers' identities.

Loosely following the logic of Rock et al. (1992), we also examined the frequency of correct flanker report for the very first flanker identification trial. ${ }^{4}$ Twenty participants $(71 \%)$ were correct in reporting the identity of the flanker on the first flanker identification trial in the 500 msec condition, and 13 participants $(46 \%)$ were correct in the 100-msec condition. The difference only approached reliability $\left[\chi^{2}(1)=3.675, .05<p<.10\right]$.

\section{Discussion}

Experiment 2 was designed to examine the extent to which flanker recall reflects the attentional processing of flankers' identities on most trials. Flanker recall after Block 1 in Experiment 2 was similar to recall at the end of Experiment 1 in terms of level and pattern of flanker recall in the 500- and 100-msec conditions; flanker recall was higher for the 500-msec duration but was reliable in both. In addition, the magnitude of the FVE for Block 1 was similar at both durations to that found in Experiment 1 .

Flanker identification, the on-line measure of attentional processing of the flankers' identities, revealed no difference in on-line reportability of flankers displayed for 500 versus $100 \mathrm{msec}$; in addition, the participants at both durations correctly reported the flankers' identities on nearly $75 \%$ of the flanker identification trials. This finding could be interpreted as showing that flanker recall actually underestimates attentional processing of the flankers' identities, especially for the shorter duration. However, the participants were slower in Block 2 than in Block 1, suggesting that their strategies may have changed.

One of the drawbacks of employing an on-line measure of processing of flanker identity is that assessing participants' knowledge of the flankers' identities might make the flankers more salient and, thus, lead to a shift in the participants' strategies. Although we took steps to reduce the likelihood of strategy changes contaminating our flanker identification measure, the fact that we assessed flanker recall and described the upcoming flanker identification trials between Block 1 and Block 2 may have led the participants to try to "pay more attention" to the flankers. The data suggest that, in fact, assessing flanker identification does change the nature of processing in the correlated flanker task. First, the data showed that overall response times were roughly $60 \mathrm{msec}$ slower during Block 2 than during Block 1 . In the standard conditions run in our lab (see Schmidt \& Dark, 1998) and in Miller's lab (see Miller, 1987, Experiment 2), the participants generally get faster from the first half to the second half of the experiment. The participants in Experiment 2 may have slowed down during Block 2, in order to figure out what the flankers were, in case they were asked to report them. Second, the magnitude of the FVE depended on whether flanker report was being assessed: The FVE was larger in Block 2 than in Block 1, suggesting that the increase in attentional processing of the flankers' identities increased the magnitude of the FVE (cf. Paquet \& Lortie, 1990). One way to avoid strategy changes would have been to assess flanker identification on a single unexpected trial (cf. Rock et al., 1992); however, such a procedure is very resource demanding and,

Table 3

Mean Proportion Correct Flanker Identification With Its Standard Deviation for Invalid and Valid Trials as a Function of Duration in Experiment 2

\begin{tabular}{llllll}
\hline & \multicolumn{4}{c}{ Trial Type } \\
\cline { 2 - 3 } \cline { 5 - 6 } Condition & \multicolumn{2}{c}{ Invalid } & & \multicolumn{2}{c}{ Valid } \\
\cline { 2 - 3 } \cline { 5 - 6 } & $M$ & $S D$ & & $M$ & $S D$ \\
\hline $500-\mathrm{msec}$ & .75 & .24 & & .76 & .22 \\
$100-\mathrm{msec}$ & .72 & .26 & & .74 & .25 \\
\hline
\end{tabular}


given our emphasis on the comparison of flanker identification with flanker recall, it did not seem appropriate.

Because the magnitude of the FVE after Block 1 is similar to the magnitude of the FVE in Experiment 1, we interpret the increase in the FVE between Block 1 and Block 2 as reflecting a shift in strategy rather than an incremental build-up of the FVE with time (cf. Miller, 1987, Experiment 3). To further test this interpretation, we reexamined the data from Experiment 1 . After the first 120 trials (Block 1), there was a mean FVE of $15 \mathrm{msec}$ $(S D=28.5)$ in the 500-msec condition and a mean FVE of $12 \mathrm{msec}(S D=29.4)$ in the $100-\mathrm{msec}$ condition. Although an incremental build-up of the flanker-response associations across trials might be expected, the data suggest that, by 120 trials, all of the learning already has occurred. Taken as a whole, the results suggest that flanker identification is more likely to reflect what can be known about the flankers' identities when participants are trying to process them along with the targets. Thus, the evidence points toward a shift in processing strategy, which makes interpretation of the flanker identification data and comparisons of flanker identification and flanker recall somewhat difficult.

Examination of the flanker recall data over the two experiments does suggest, however, that flanker recall reflects attentional processing of flanker identity on most trials. We base this conclusion on two observations. First, the accuracy of flanker report on the very first flanker identification trial, which might more directly reflect processing before a strategy shift, showed a pattern consistent with the flanker recall results: Correct first flanker identification was numerically higher in the $500-\mathrm{msec}$ duration than in the $100-\mathrm{msec}$ duration, and the magnitude was similar to the level of flanker recall obtained at the end of Block 1.

Second, the magnitude of flanker recall after 120 trials (after Block 1 of Experiment 2) was similar to that found after 320 trials (at the end of Experiment 1). Several reviewers have suggested that flanker recall might reflect attentional processing of flankers' identities on an atypical subset of trials. If flanker recall reflects attentional processing of the flankers' identities on trials on which the incorrect response is made to the target, there would be three times the opportunity for such trials to occur within 320 trials than within 120 trials, and, therefore, there should be higher flanker recall after more trials. If flanker recall reflects attentional processing of flankers' identities on the first few trials, before the participant has learned to focus attention, there should be higher recall over a shorter retention interval (120 vs. 320 trials). Finally, if flanker recall reflects attentional processing of flanker's identities on the last few trials, then the "last few trials" must be defined as a set of typical trials, because the participant does not know when they will occur. Thus, the data provide converging evidence that participants are aware of the flankers' identities on most trials and that flanker recall is an adequate measure of on-line flanker processing.
Our interpretation of the results of Experiment 2 is that flanker recall actually underestimates the extent to which participants can become aware of the flankers on each trial but that it is a good measure of the extent to which participants $d o$ become aware of flankers' identities in the standard correlated flanker task. The results of Experiment 2 also showed that the FVE is sensitive to changes in strategy: The magnitude of the FVE can be influenced by intentional attentional processing of flanker identity, although it is not affected by manipulation of flanker duration when flankers are supposed to be ignored.

On the surface, the data from Experiments 1 and 2 seem to suggest that the FVE does not reflect attentional processing of flankers' identities. The dissociation of the effect of flanker duration on flanker recall and on the FVE suggests that the two reflect different types of processing: Flanker recall reflects attentional processing of flankers' identities, whereas the FVE might reflect automatic processing of flankers' identities. One caveat to this conclusion is the fact that the magnitude of the FVE increased in a situation in which the participants might have been trying to process flanker identity more and, thus, is not reflecting a strongly automatic process (Kahneman \& Treisman, 1984). Moreover, although it has been demonstrated that the FVE does not depend on the level of attentional processing of the flankers that leads to flanker recall, it has not yet been demonstrated that the FVE does not depend on some minimal level of attentional processing of the flankers' identities. Experiment 3 was designed to examine this possibility.

\section{EXPERIMENT 3}

The duration manipulation employed in Experiment 1 was expected to limit attentional processing of flankers' identities in such a way that flanker recall would be eliminated, allowing the FVE to be measured under conditions in which there was no evidence of attentional processing of flankers' identities. The briefest duration, however, still yielded above-chance flanker recall, allowing for the possibility that some minimal amount of attentional processing of the flankers' identities is needed for the FVE. In order to adequately test this possiblitity, in Experiment 3 , we examined whether the FVE would obtain under conditions in which participants were unaware of the flankers' identities, as measured by flanker recall. To this end, we employed brief flanker durations (viz., 200, 100 , and $50 \mathrm{msec}$ ), with flankers that were either masked or unmasked.

The purpose of Experiment 3 was to explore the boundary conditions under which both flanker recall and the FVE are obtained. We expected that the combination of brief durations and masking would produce such datalimited displays that both flanker recall and the FVE would be eliminated at some point. Again, as in Experiment 1 , the question was whether there was evidence of a relationship between flanker recall, a measure of attentional processing of flanker identity, and the FVE. In 
general, we were interested in determining under what condition(s) flanker recall and the FVE would be eliminated. In particular, the question was whether the FVE would obtain under conditions that do not provide the minimal amount of attentional processing of the flankers needed to produce above-chance flanker recall. If awareness of the flankers' identities is necessary for the FVE, no reliable FVE should be found when the participants cannot reliably recall any of the flankers.

\section{Method}

\section{Participants}

One hundred sixty-eight right-handed students (55 male and 113 female), from the same pool as that used in Experiments 1 and 2, received extra credit in an undergraduate psychology course for participating in this experiment. The participants were randomly assigned to one of the six conditions: masked and unmasked at each of three durations $(200,100$, and $50 \mathrm{msec})$. Each participant was tested in a single session lasting approximately $45 \mathrm{~min}$. The data from 11 participants were replaced because of low target-response accuracy (less that $85 \%$ correct). One participant was replaced in the 200-msec unmasked condition, 1 in the 100 -msec masked conditions, 2 in the $100-\mathrm{msec}$ unmasked condition, 2 in the 50 -msec unmasked condition, 2 in the 50-msec masked conditions, and 3 in the 200-msec masked condition.

\section{Procedure}

The stimuli, apparatus, and procedure for Experiment 3 were identical to those of Experiment 1, except that different flanker durations were used and, for half of the conditions, flankers were masked. Masking was accomplished by replacing flankers with a \# symbol, instead of a blank, when flanker duration expired. The unmasked conditions were identical to their counterparts in Experiment 1. All other aspects of the two experiments were the same.

\section{Results}

An alpha level of .05 was used for all analyses.

\section{The Flanker Validity Effect}

A separate mean response time was computed for valid and for invalid trials for each participant; only accurate response times were included. The group mean response times and proportions correct for valid and invalid trials in the six conditions are shown in Table 4. In all conditions, target-response accuracy was above $95 \%$

Table 4

Mean Response Time (in Milliseconds) With Its Standard Deviation and Proportion Correct for Invalid and Valid Trials as a Function of Duration and Masking in Experiment 3

\begin{tabular}{|c|c|c|c|c|c|c|}
\hline \multirow[b]{3}{*}{ Condition } & \multicolumn{6}{|c|}{ Trial Type } \\
\hline & \multicolumn{3}{|c|}{ Invalid } & \multicolumn{3}{|c|}{ Valid } \\
\hline & $M$ & $S D$ & PC & $M$ & $S D$ & PC \\
\hline \multicolumn{7}{|l|}{$200-\mathrm{msec}$} \\
\hline Unmasked & 514 & 71 & .96 & 510 & 73 & .97 \\
\hline Masked & 517 & 59 & .96 & 513 & 58 & .96 \\
\hline \multicolumn{7}{|l|}{$100-$ msec } \\
\hline Unmasked & 557 & 73 & .95 & 548 & 72 & .97 \\
\hline Masked & 535 & 44 & .97 & 525 & 42 & .97 \\
\hline \multicolumn{7}{|l|}{50 -msec } \\
\hline Unmasked & 543 & 84 & .96 & 537 & 81 & .96 \\
\hline Masked & 528 & 67 & .96 & 521 & 66 & .97 \\
\hline
\end{tabular}

correct. An ANOVA on response times, with duration and masking as between-subjects variables and validity as a within-subjects variable, showed a reliable effect of validity $\left[F(1,162)=24.68, M S_{\mathrm{e}}=136.5\right]$, with faster response times for valid trials. The effect of duration approached reliability $\left[F(1,162)=2.55, M S_{\mathrm{e}}=8,837.0\right.$, $.05<p<.10$ ], with a tendency for shorter response times in the 200-msec conditions. The interaction effect was not reliable.

The FVE is plotted as a function of duration and masking in Figure 3. The mean FVE from each condition was compared with zero, to determine whether the observed FVE was reliably greater than chance. The FVE was reliable in both the 100- and the 50-msec masked and unmasked conditions, but not in the $200-\mathrm{msec}$ masked and unmasked conditions.

\section{Flanker Recall}

The mean proportions of flankers correctly recalled is shown in Figure 4 as a function of duration and masking. An ANOVA, with duration and masking as betweensubjects variables, showed a reliable effect of masking $\left[F(1,162)=10.24, M S_{\mathrm{e}}=0.08\right]$, with higher recall for unmasked flankers, and a reliable effect of duration $\left[F(2,162)=6.13, M S_{\mathrm{e}}=0.08\right]$, but the interaction effect was not reliable. A planned linear contrast on duration collapsed over masking was reliable $[F(1,162)=$ $\left.11.78, M S_{\mathrm{e}}=0.08\right]$, whereas the residual sum of squares was not, showing that, as flanker duration increased, the level of flanker recall also increased.

Flanker recall in all six conditions also was compared with chance-level recall $(.18)$ in order to determine whether awareness had been sufficiently limited by the brief durations and masking. Flanker recall was above chance in every condition except the 50 -msec masked condition. Thus, only under the most extreme data-limited conditions was awareness of the flankers' identities eliminated.

\section{Discussion}

Experiment 3 was designed to assess whether the FVE would be reliable under conditions in which there was little evidence of attentional processing of flanker identity, as reflected in chance-level flanker recall. The duration and masking manipulations were successful at limiting awareness of the flankers' identities. As was expected, the level of flanker recall decreased as flanker duration decreased, and the level with masked flankers was lower than the level with unmasked flankers. Unexpectedly, however, flanker recall remained above chance in all but the 50 -msec masked condition. The fact that reliable flanker recall is obtained under even very datalimited conditions suggests that flanker recall is, in fact, a rather sensitive measure of the amount of attentional processing of the flankers' identities.

The FVE did not vary reliably as a function of either duration or masking, but the magnitude of the FVE was not above chance in either the 200-msec unmasked or the $200-\mathrm{msec}$ masked condition. We have no explanation for 


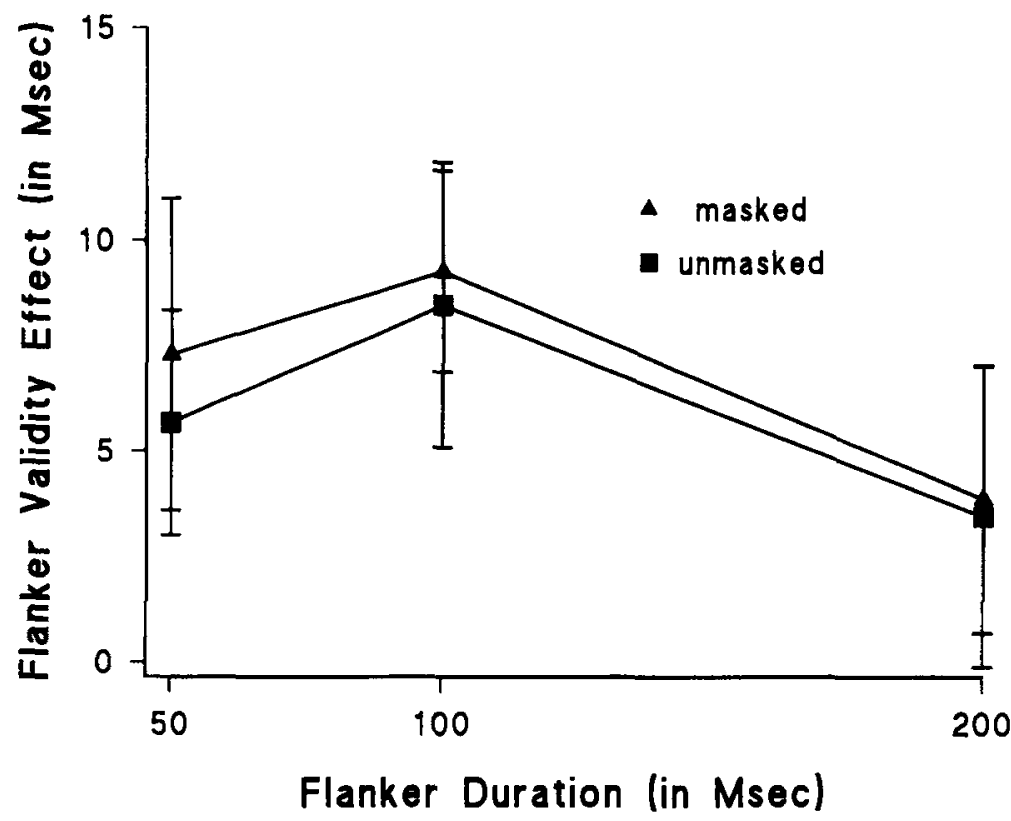

Figure 3. Mean flanker validity effect (in milliseconds) with standard error as a function of flanker duration and masking in Experiment 3.

why the FVE with 200-msec flankers appears to be weaker than in the other conditions. We note, however, that a similar pattern was apparent in Experiment 1.

The results of Experiment 3 demonstrated dissociations in both the effect of duration and the effect of masking on the FVE and on flanker recall: The FVE did not vary as a function of flanker duration, and it did not vary as a function of masking, whereas flanker recall decreased monotonically as a function of duration and was lower for masked than for unmasked flankers. The data provide converging evidence that the FVE does not depend on participants' awareness of the flankers' identities. The dissociations further support the idea that more attentional processing of the flankers' identities does not increase the FVE under standard ignore-the-flankers instructions.

The test of whether there is a minimal amount of attentional processing of the flankers' identities needed to produce the FVE is provided by the 50 -msec masked

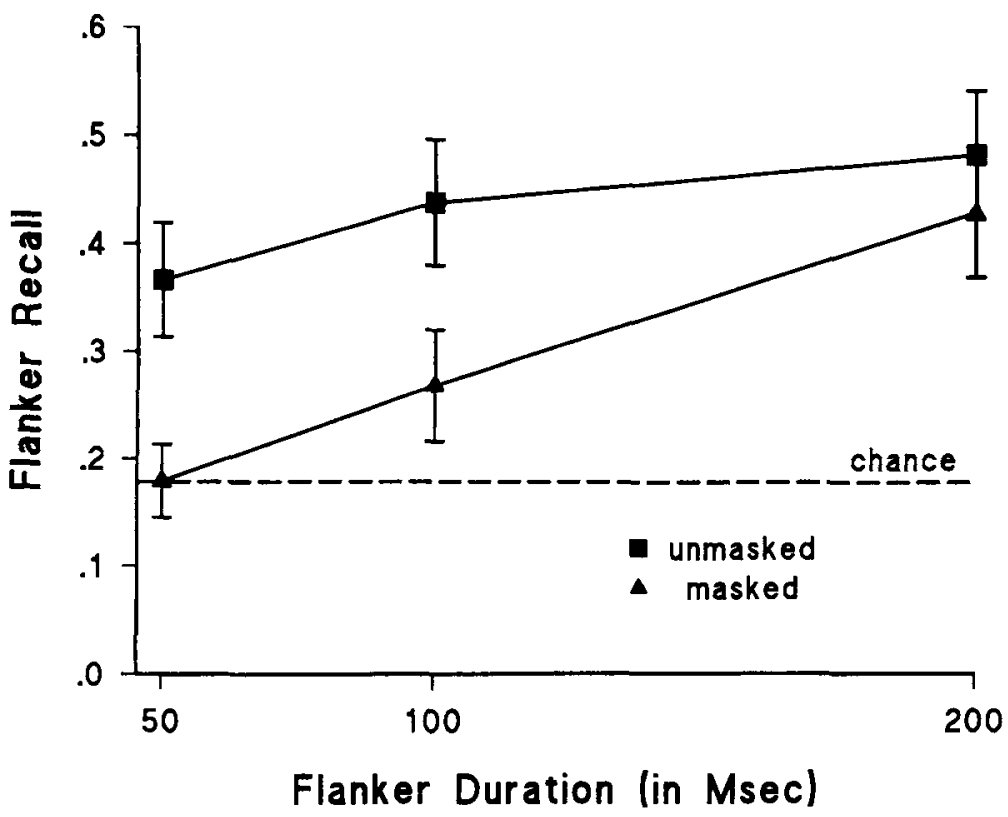

Figure 4. Mean proportion correct flanker recall with standard error as a function of flanker duration and masking in Experiment 3. 
condition. In that condition, there was chance-level flanker recall, but there was a reliable FVE. Thus, the data suggest that the minimal amount of attentional processing of the flankers that leads to long-term memory for flanker identity is not needed to support the FVE. Not only was the FVE reliable in the 50-msec masked condition, it was of a similar magnitude to those found in other conditions. We note that similar results have been reported with the Eriksen flanker task: Schwarz and Mecklinger (1995) found a reliable flanker effect for flankers that were presented below the threshold of awareness.

\section{EXPERIMENT 4}

The main purpose of Experiment 3 was to determine whether the FVE depends on some minimal level of attentional processing of the flankers' identities. The results suggested that the amount of attentional processing of flankers' identities that is required to produce abovechance flanker recall is not required for the FVE. Of course, above-chance flanker recall was demonstrated in all but the most extreme data-limited condition. The 50msec masked condition is the only condition in a wide variety of flanker tasks in our lab in which we have not found reliable flanker recall co-occurring with the FVE.

The primary purpose of Experiment 4 was to replicate the $50-\mathrm{msec}$ masked condition, to see whether the same pattern of results would obtain. Also of interest was whether, under the 50-msec masked condition, the participants would be able to become aware of the flankers, if they chose to do so. Thus, Experiment 4 employed the procedure developed in Experiment 2 to examine performance with $50-\mathrm{msec}$ masked flankers.

\section{Method}

\section{Participants}

Twenty-six right-handed students, selected from the same participant pool as that in the previous experiments, received extra credit for participating in this experiment. Each participant was tested in a single session lasting approximately $35 \mathrm{~min}$.

\section{Procedure}

The stimuli, apparatus, and procedure for Experiment 4 were identical to those for Experiment 2, except that flanker duration was $50 \mathrm{msec}$ and flankers were masked, as in Experiement 3 . Thus, the general procedure included a block of 120 standard trials (Block 1 ), after which flanker recall was assessed, followed by a block of 240 trials (Block 2), in which flanker identification was assessed. In addition, after Block 2, flanker recall was assessed for a second time.

\section{Results}

An alpha level of .05 was used for all analyses.

\section{Flanker Validity Effect}

A separate mean response time was computed for valid and for invalid trials for each participant; only accurate response times were included. The group mean response times and proportions correct for valid and invalid trials as a function of block are shown in Table 5. Target-response accuracy was above $91 \%$ correct in
Table 5

Mean Response Time (in milliseconds) With Its Standard Deviation and Proportion Correct for Invalid and Valid Trials as a Function of Block in Experiment 4

\begin{tabular}{cccccccc}
\hline & \multicolumn{5}{c}{ Trial Type } \\
\cline { 2 - 8 } Condition & \multicolumn{3}{c}{ Invalid } & & \multicolumn{3}{c}{ Valid } \\
\cline { 2 - 5 } \cline { 5 - 8 } 50-msec Masked & $M$ & $S D$ & PC & & $M$ & $S D$ & PC \\
\hline Block 1 & 529 & 62 & .95 & & 521 & 65 & .96 \\
Block 2 & 656 & 154 & .91 & 647 & 139 & .93 \\
\hline
\end{tabular}

both blocks. An ANOVA on response times, with block and validity as within-subjects variables, showed a reliable effect of block $\left[F(1,25)=26.76, M S_{\mathrm{e}}=15,514.8\right]$, with faster response times for Block 1 , and a reliable effect of validity $\left[F(1,25)=7.73, M S_{\mathrm{e}}=274.3\right]$, with faster response times for valid trials. There was not a reliable block $\times$ validity interaction effect $(F<1)$. The FVE was computed for each block and compared with zero, to determine whether the observed FVE was reliably greater than chance; it was reliable for both for Block 1 and Block 2.

\section{Knowledge of Flanker Identity}

Flanker recall. The mean proportions of flankers correctly recalled after Block 1 was .16 $(S D=.22)$ and after Block 2 was $.64(S D=.35)$. A comparison of the means revealed lower flanker recall after Block 1 than after Block 2. Flanker recall was not above chance (.18) after Block 1, but it was above chance after Block 2.

Flanker identification. The mean proportion of correct flanker identification in Block 2 was $.38(S D=.26)$ for valid test trials and $.31(S D=.25)$ for invalid test trials; a comparison of the means showed higher report for valid test trials. Flanker identification was greater than chance (.18) for both valid and invalid test trials.

The relationship between flanker identification and Block 2 flanker recall (recall at the end of Experiment 4) also was examined. Of the possible four flankers to be reported, the mean proportion of specific flankers identified across the 32 test trials was .70-that is, on average, the participants identified 2.8 of the 4 flankers. Comparison of specific flankers identified with specific flankers recalled revealed that the proportion of reported flankers that were not subsequently recalled was only .05 . Finally, the proportion of flanker letters that were recalled but had not been identified on line was only .17 - that is, nonidentified flankers were recalled at chance level.

We also examined the frequency of correct flanker identification for the very first flanker identification trial. Nine participants $(35 \%)$ were correct in reporting the flanker on the first identification trial.

\section{Discussion}

Experiment 4 was designed to assess the degree to which participants could become aware of the flankers' identities under conditions that had not shown reliable flanker recall in Experiment 3. The data from Block 1 
basically mirrored the findings of Experiment 3: There was a reliable FVE, but flanker recall was at chance. A reliable FVE can be obtained with little evidence of attentional processing of the flankers' identities.

As in Experiment 2, there was a slowing of responses in Block 2, suggesting that there may have been a strategy shift such that the participants may have been trying to see more of the displays when there was the possibility that flanker identification would be requested. The flanker identification data suggest that, even with 50 msec masked flankers, participants can become aware of the flankers' identities; however, we are not arguing that they became aware of flankers' identities in Block 1 . With these extreme data-limited displays, participants do not seem to do enough attentional processing of the flankers' identities under standard conditions to become aware of them. The fact that participants can become aware of the flankers' identities immediately after responding to a target, however, does suggest that flankers' identities may be undergoing some attentional processing. The data do not provide information about whether this processing is related to the FVE.

\section{GENERAL DISCUSSION}

The results of Experiment 1 revealed a functional dissociation between flanker recall and the FVE: Flanker recall decreased monotonically as a function of decreased flanker duration, whereas the FVE did not vary. Functional dissociations frequently have been interpreted as revealing the operation of independent processes (e.g., Jacoby, Lindsay, \& Toth, 1992). Thus, the dissociation could be interpreted as evidence that the FVE reflects automatic processing of the flankers' identities, whereas flanker recall reflects attentional processing. Before the FVE could be fully dissociated from attentional processing of flankers' identities, however, it was necessary to consider (1) whether flanker recall is an adequate measure of flanker processing on most trials and (2) whether there is a minimal amount of attentional processing of flanker identities necessary for the FVE. The results of Experiment 2 suggested that, although flanker recall may underestimate participants' ability to become aware of the flankers' identities if they choose to do so, there does seem to be attentional processing of the flankers on most trials, and flanker recall seems to be a sensitive measure of that processing. Experiment 2 also showed that the magnitude of the FVE can be increased by emphasizing the flankers; this suggests that the FVE does not reflect a strongly automatic process (cf. Kahneman \& Treisman, 1984). Experiment 3 attempted to examine the boundary conditions for both flanker recall and the FVE. The boundary was reached at which there was no flanker recall, but flanker validity remained constant and reliable under most conditions. Experiment 4 replicated the finding of a FVE, with no evidence of attentional processing of the flankers' identities.
When Miller (1987) introduced the correlated flanker task, he offered a late-selection explanation of the FVE. He stated that flankers undergo only automatic processing, at least while the target is being processed, and that the processing includes the identity of the flankers. As a result of repetitive automatic activation of flankers' identities in close temporal relationship to the execution of specific responses, an association is formed between the flanker identities and the responses. The learning, like the processing of flanker identities, is automatic. Miller (1987) found no evidence that participants could recall the flankers' identities, and he used that fact to support the conclusion that participants are not aware of what they are learning or even that they are learning.

Miller's (1987) view predicts a dissociation in the effect of attentional manipulations (such as duration and masking) on flanker recall and the FVE and, thus, might be offered as a good explanation of the current data. However, Miller's (1987) view depends on there being no evidence of attentional processing of the flankers' identities. The empirical support for his view was challenged by the findings of Schmidt and Dark (1998), who obtained high flanker recall with the correlated flanker task. Across experiments, Schmidt and Dark modified their procedure, bringing it closer and closer to a replication of Miller's procedure, and concluded that it was likely that the difference in the recall levels was the result of differences in participants' response criteria. Miller (1987) allowed participants to say that they could not recall any flankers, and, in fact, that was the most frequent response, whereas Schmidt and Dark required participants to make some recall response. Schmidt and Dark concluded that there was a failure of selective attention in the correlated flanker task and offered an earlyselection view of the FVE. Flanker identities undergo attentional processing, and it is likely that this processing is responsible for the FVE.

The current results require a modification of the conclusions of Schmidt and Dark (1998). Although flanker recall does show that there is a failure of selective attention in the correlated flanker task, the FVE does not depend on the same type of attentional processing of the flankers' identities as that which leads to recall. It is likely that whenever attention is focused on some location, stimuli at that location receive attentional processing, even if such processing is insufficient to produce awareness of the identity of the stimulus (e.g., the 50-msec masked condition). It may be this attentional processing that underlies the FVE.

This modified view has aspects in common with Fournier (1994). Fournier suggested that there is a failure of selective attention in flanker tasks because targets and flankers appear simultaneously as abrupt onsets and in close proximity. As a result, flankers and targets initially are perceived and attended to as a single object. When this occurs, it is necessary to utilize a segregation process to perceptually separate the to-be-responded-to target from the flankers. The segregation process might in- 
hibit flanker processing, so that only the target receives further attentional processing. On this account, our data suggest that the original attentional processing is sufficient to produce the FVE and also, except under extremely data-limited conditions, to produce memory traces that include information about flanker identities that can be used during flanker recall. Although processing of flanker identity could be part of the presegregation attentional processing, we see no reason that this must be the case. The FVE does not depend on knowledge of flankers' identities, even though the identities typically are available in memory. Thus, we wish to suggest the possibility that the FVE, at least with target displays of $500 \mathrm{msec}$ or less, does not even depend on the processing of flankers' identities. Perhaps the FVE simply reflects the impact of practice in processing and responding to specific displays. Perhaps responses to valid trials are faster because they are more automatized (Logan, 1988).

Miller (1987, Experiment 2) considered the possibility that frequency of display could be responsible for the difference in response times to valid and invalid trials. He found that targets presented equally often with specific flankers were still responded to more quickly when they were presented with a flanker that was correlated (through another target) with the appropriate response than when they were presented with a flanker correlated with the wrong response. This difference could not be explained in terms of display frequency. Although we acknowledge this finding, we point out that there were two anomalous findings in the data. First, there was a reliable, but unexplained, difference in the speed with which responses were made to the two classes of targets. Second, there was an interaction between hand of response and the FVE for those targets through which validity was established (the more frequent displays). Thus, no unequivocal conclusion could be drawn about the effect of display frequency on response time. We currently are exploring the relationship between display frequency and the FVE.

Past research has suggested that there is a failure of selective attention at some point in processing in the correlated flanker task such that flankers are attended to $(\mathrm{Pa}-$ quet \& Lortie, 1990; Schmidt \& Dark, 1998), and the current results support that view. The purpose of the current research was to examine the role of attentional processing of the flankers' identities in the FVE. Across four experiments, we manipulated flanker duration and masking in order to limit the amount of attentional processing of the flankers' identities. Both duration and masking affected level of flanker recall: The less information that was provided by the environment, the less information there was that was available at the time of recall. For the most part, the manipulations did not affect the magnitude of the FVE. Our previous research (see, also, Carlson \& Flowers, 1996; Miller, 1987) showed that participants lack explicit knowledge of the flanker-response correlations, suggesting that attentional processing of the relationships is not necessary for the FVE. The current research shows that awareness of the flankers' identities is not related to the magnitude of the FVE and is not even necessary for the FVE to obtain. But how is this different from the point originally made by Miller (1987)? Although the results support Miller's (1987) conclusion that there is no relationship between awareness of flankers' identities and the FVE, they do not support his conclusion that flankers' identities are processed automatically (i.e., without attention). Rather, the results suggest an even stronger conclusion: The FVE does not even depend on flanker identity. We would argue that, because there is a failure of selective attention in the correlated flanker task, the dissociations reported in the current research suggest that flanker recall and the FVE reflect different attentional processes.

\section{REFERENCES}

Carlson, K. A., \& Flowers, J. H. (1996). Intentional versus unintentional use of contingencies between perceptual events. Perception $\&$ Psychophysics, 58, 460-470.

COHEN, J. (1988). Statistical power analyses for the behavioral sciences. Hillsdale, NJ: Erlbaum.

CowaN, N. (1988). Evolving conceptions of memory storage, selective attention, and their mutual constraints within the human informationprocessing system. Psychological Bulletin, 104, 163-191.

ERIKSEN, B. A., \& ERIKSEN, C. W. (1974). Effects of noise letters upon the identification of a target letter in a nonsearch task. Perception $\&$ Psychophysics, 16, 143-149.

ERIKSEN, C. W., \& HoffMan, J. E. (1972). Temporal and spatial characteristics of selective encoding from visual displays. Perception \& Psychophysics, 12, 201-204.

ERIKSEN, C. W., \& Hoffman, J. E. (1973). The extent of processing of noise elements during selective encoding from visual displays. Perception \& Psychophysics, 14, 155-160.

FISK, A. D., \& SCHNEIDER, W. (1984). Memory as a function of attention, level of processing, and automatization. Journal of Experimental Psychology: Learning, Memory, \& Cognition, 10, 181-197.

FourniER, L. R. (1994). Selective attentional delays and attentional capture among simultaneous visual onset elements. Perception \& Psychophysics, 56, 536-550.

HARMS, L., \& BundeSEN, C. (1983). Color segregation and selective attention in a nonsearch task. Perception \& Psychophysics, 33, 11-19.

JACOBY, L.L., LINDSAY, D. S., \& ToTH, J. P. (1992). Unconscious influences revealed: Attention, awareness, and control. American Psychologist, 47, 802-809.

Johnston, W. A., \& DARK, V. J. (1986). Selective attention. Annual Review of Psychology, 37, 43-75.

KaHNEMAN, D., \& TrelSMAN, A. (1984). Changing views of attention and automaticity. In R. Parasuraman \& D. R. Davies (Eds.), Varieties of attention (pp. 29-61). New York: Academic Press.

Kramer, A. F., \& Jacobson, A. (1991). Perceptual organization and focused attention: The role of objects and proximity in visual processing. Perception \& Psychophysics, 50, 267-284.

LOGAN, G. D. (1988). Toward an instance theory of automatization. Psychological Review, 95, 492-527.

Miller, J. (1987). Priming is not necessary for selective-attention failures: Semantic effects of unattended, unprimed letters. Perception \& Psychophysics, 41, 419-434.

MilleR, J. (1991). The flanker compatibility effect as a function of visual angle, attentional focus, visual transients, and perceptual load: A search for boundary conditions. Perception \& Psychophysics, 49, 270-288.

PAQuet, L., \& LorTIE, C. (1990). Evidence for early selection: Precuing target location reduces interference from same-category distractors. Perception \& Psychophysics, 48, 382-388. 
Rock, I., Linnett, C. M., Grant, P., \& Mack, A. (1992). Perception without attention: Results of a new method. Cognitive Psychology, 24, 502-534.

ROEDIGER, H. L., III (1990). Implicit memory: Retention without remembering. American Psychologist, 45, 1043-1056.

SchmidT, P. A., \& DARK, V. J. (1998). Attentional processing of "unattended" flankers: Evidence for a failure of selective attention. Perception \& Psychophysics, 60, 227-238.

SCHNEIDER, W. (1988). Micro Experimental Laboratory: An integrated system for IBM PC compatibles. Behavior Research Methods, Instruments, \& Computers, 20, 206-217.

Schwarz, W., \& Mecklinger, A. (1995). Relationship between flanker identifiability and compatibility effect. Perception \& Psychophysics, 57, 1045-1052.

SHIFFrin, R. M., Diller, D., \& CoHEN, A. (1996). Processing visual information in an unattended location. In A. F. Kramer, M. G. H. Coles, \& G. D. Logan (Eds.), Converging operations in the study of visual selective attention (pp. 225-245). Washington, DC: American Psychological Association.

SHIfFrIN, R. M., \& SCHNEIDER, W. (1977). Controlled and automatic human information processing: II. Perceptual learning, automatic attending, and a general theory. Psychological Review, 84, 127-190.

Stadler, M. A., \& Proctor R. W. (1993, November). Attention and covariation learning in the Eriksen flanker task. Paper presented at the 34th Annual Meeting of the Psychonomic Society, Washington, DC.
YANTIS, S., \& Johnston, J. C. (1990). On the locus of visual selection: Evidence from focused attention tasks. Journal of Experimental Psychology: Human Perception \& Performance, 16, 135-149.

\section{NOTES}

1. Following Schmidt and Dark (1998), we also assessed participants' knowledge of the flanker-response correlatons with three tasks (flanker assignment, valid display estimation, and novel target accuracy). Like Miller (1987) and Schmidt and Dark, we found no evidence that participants had any explicit knowledge of the flanker-response correlations.

2. We than Jeff Miller for pointing out this possibility to us.

3. We thank Asher Cohen and Arthur Kramer for pointing out this possibility to us.

4. Rock et al. (1992) assessed awareness under conditions in which participants had no idea that they would be asked about the unattended stimuli. Although the flanker identification task had already been described to the participants, we reasoned that their processing strategy might not change until after the actual task changed (not just the instructions); thus, the first flanker identification trial might be somewhat surprising and might be a good estimate of processing before any changes in strategy.

(Manuscript received February 10, 1997; revision accepted for publication March 1, 1998.) 